\title{
MAXIMAL AUTOMORPHISMS OF CALABI-YAU MANIFOLDS VERSUS MAXIMALLY UNIPOTENT MONODROMY
}

\author{
JAN CHRISTIAN ROHDE
}

\begin{abstract}
Let $\alpha$ be an automorphism of the local universal deformation of a CalabiYau 3-manifold $X$, which does not act by \pm id on $H^{3}(X, \mathbb{C})$. We show that $X$ can not be a fiber of a maximal family with maximally unipotent monodromy, if such an automorphism $\alpha$ exists. Moreover we classify the possible actions of $\alpha$ on $H^{3}(X, \mathbb{C})$, construct examples and show that the period domain is a complex ball containing a dense set of $C M$ points in this case.
\end{abstract}

\section{INTRODUCTION}

Due to their importance in theoretical physics, we are interested in Calabi-Yau 3manifolds. We consider the automorphisms of Calabi-Yau 3-manifolds, which extend to automorphisms of the local universal deformation. Here such automorphisms are called maximal.

An automorphism of a $K 3$ surface $S$ corresponds to a Hodge isometry on $H^{2}(S, \mathbb{Z})$ and can be studied in terms of isometries of this integral lattice (see [1], [13, [16]). In the case of a Calabi-Yau 3-manifold $X$ there can be automorphisms acting trivially on $H^{3}(X, \mathbb{Z})$. However, the actions of a maximal automorphism $\alpha$ of $X$ on $H^{3}(X, \mathbb{Z})$ and the variations of Hodge structures of the families containing $X$ as fiber are studied here. We show that a maximal automorphism $\alpha$ forces the $F^{2}$-bundle to be constant in the $V H S$ of a maximal family (i. e. a family such that the Kodaira-Spencer map is an isomorphism at every point), if $\alpha$ does not act as \pm id on $H^{3}(X, \mathbb{Z})$. In this case $\alpha$ forbids $X$ to be a fiber of a maximal family with maximally unipotent monodromy. Thus the assumptions of a formulation of the mirror conjecture in 12 can not be satisfied by $X$ in this case. We show that in this case the period domain is a complex ball and each maximal family containing $X$ as fiber has a dense set of complex multiplication $(C M)$ fibers. Theoretical physicists are interested in Calabi-Yau 3-manifolds with $C M$ - in particular if there exists a mirror pair of Calabi-Yau 3-manifolds with $C M$ (see [10]).

Moreover we will see that the quotient $Q(X)$ of the maximal automorphisms of $X$ by the automorphisms acting trivially on $H^{3}(X, \mathbb{Z})$ is given by

$$
\{e\}, \mathbb{Z} /(2), \quad \mathbb{Z} /(3), \quad \mathbb{Z} /(4) \text { or } \mathbb{Z} /(6) .
$$

For each $0 \leq k \leq 6$ we construct simply-connected Calabi-Yau 3-manifolds $X$ with $Q(X) \supseteq \mathbb{Z} /(3)$ and the Hodge numbers

$$
h^{2,1}(X)=6-k \text { and } h^{1,1}(X)=18+11 \cdot k .
$$

This paper was written at the Graduiertenkolleg "Analysis, Geometrie und Stringtheorie" at Leibniz Universität Hannover. I would like to thank Klaus Hulek very much for many stimulating discussions and his hint to the article [5], which yields the construction method of the examples with $Q(X) \supseteq \mathbb{Z} /(3)$. Moreover I would like to thank Bernd Siebert. He has made me aware about the action of an automorphism of a Calabi-Yau manifold on its local universal deformation and read an early version of this paper. I would like to thank my former doctoral adviser Eckart Viehweg for looking over this text. 


\section{Contents}

Introduction

1. Some conventions, notations and facts

2. The actions of automorphisms on complex structures

3. The cohomology actions of maximal automorphisms

4. The construction of examples

5. Consequences for the monodromy

6. Consequences for complex multiplication

References

\section{Some COnVEntions, Notations And FACTS}

We follow the conventions and notations of [14]. Let $V$ be a finitely generated $\mathbb{Z}$-module,

$$
S^{1}=\operatorname{Spec}\left(\mathbb{R}[x, y] /\left(x^{2}+y^{2}-1\right)\right) \text { with } S^{1}(\mathbb{R}) \cong\{z \in \mathbb{C} \mid z \bar{z}=1\}
$$

and $h: S^{1} \rightarrow \mathrm{GL}\left(V_{\mathbb{R}}\right)$ be a homomorphism of $\mathbb{R}$-algebraic groups. Each integral Hodge structure of some fixed weight $k$ can be given by a pair $(V, h)$. The $\operatorname{Hodge~group~} \operatorname{Hg}(V, h)$ is the smallest $\mathbb{Q}$-algebraic subgroup $G$ of $\mathrm{GL}\left(V_{\mathbb{Q}}\right)$ such that $h\left(S^{1}\right) \subset G_{\mathbb{R}}$.

1.1. Each integral Hodge structure has the trivial automorphisms given by \pm id. Here an automorphism $\alpha$ of a compact Kähler manifold $X$ of dimension $n$ is denoted as a translation, if it acts as id on $H^{n}(X, \mathbb{Z})$. Moreover $\alpha$ is a super-translation, if it acts as a trivial automorphism on the Hodge structure $\left(H^{n}(X, \mathbb{Z}), h_{X}\right)$.

Note that the action of the automorphisms of $X$ on $H^{n}(X, \mathbb{Z})$ yields a group homomorphism $f: \operatorname{Aut}(X) \rightarrow \operatorname{Aut}\left(H^{n}(X, \mathbb{Q}), h_{X}\right)$. Its kernel is given by the translations. Thus the translations yield a normal subgroup of $\operatorname{Aut}(X)$. Note that \pm 1 are the only integers $z$ such that the multiplication by $z$ fixes the polarization on $H^{n}(X, \mathbb{Z})$, if $H^{n}(X, \mathbb{Z}) \neq 0$. Hence the kernel of the composition of $f$ with the natural map $\operatorname{Aut}\left(H^{n}(X, \mathbb{Q}), h_{X}\right) \rightarrow \operatorname{PGL}\left(H^{n}(X, \mathbb{Q})\right)$ is given by the super-translations. Thus the super-translations yield a normal subgroup of $\operatorname{Aut}(X)$, too.

Here a Calabi-Yau 3-manifold $X$ is a compact Kähler manifold of dimension 3 such that

$$
\omega_{X} \cong \mathcal{O}_{X} \text { and } h^{k, 0}(X)=0 \text { for } k=1,2 .
$$

From now on let $X$ denote a Calabi-Yau 3-manifold.

1.2. The Calabi-Yau 3-manifold $X$ has the following intermediate Jacobians (see [3]):

- The Griffiths intermediate Jacobian $\mathrm{J}_{G}(X)$ is the complex torus corresponding to the Hodge structure of type $(1,0),(0,1)$ on $H^{3}(X, \mathbb{Z})$ given by

$$
H^{1,0}:=H^{3.0}(X) \oplus H^{2,1}(X), \quad H^{0,1}:=H^{1,2}(X) \oplus H^{0,3}(X) .
$$

- The Weil intermediate Jacobian $\mathrm{J}_{W}(X)$ is the abelian variety corresponding to the Hodge structure of type $(1,0),(0,1)$ on $H^{3}(X, \mathbb{Z})$ given by

$$
H^{1,0}:=H^{2,1}(X) \oplus H^{0,3}(X), \quad H^{0,1}:=H^{3,0}(X) \oplus H^{1,2}(X) .
$$

It is a well-known fact that one can decompose rational Hodge structures into simple rational Hodge structures, i. e. indecomposable rational Hodge structures. Let $\left(H_{S}^{3}(X, \mathbb{Q}), h_{S}\right)$ denote the simple rational sub-Hodge structure of $\left(H^{3}(X, \mathbb{Q}), h_{X}\right)$ which satisfies

$$
H^{3,0}(X) \subset \underset{2}{H_{S}^{3}}(X, \mathbb{C})
$$


Moreover let $\operatorname{End}_{\mathbb{Q}}\left(H_{S}^{3}(X, \mathbb{Q}), h_{S}\right)$ denote the algebra of automorphisms on $\left(H_{S}^{3}(X, \mathbb{Q}), h_{S}\right)$ commuting with $\operatorname{Hg}\left(H_{S}^{3}(X, \mathbb{Q}), h_{S}\right)$.

Lemma 1.3. The action of $\operatorname{End}_{\mathbb{Q}}\left(H_{S}^{3}(X, \mathbb{Q}), h_{S}\right)$ on $H^{3,0}(X)$ yields an isomorphism

$$
\operatorname{End}_{\mathbb{Q}}\left(H_{S}^{3}(X, \mathbb{Q}), h_{S}\right) \rightarrow \mathbb{F} \subset \mathbb{C}
$$

of rings, where $\mathbb{F}$ is a number field.

Proof. (see [3])

Lemma 1.4. Each automorphism $\alpha$ of $X$ acts on $H^{3,0}(X)$ as the multiplication with a root of unity.

Proof. By the assumptions, $\operatorname{dim} H^{3,0}(X)=1$ and $\alpha\left(H^{3,0}(X)\right)=H^{3,0}(X)$. Thus $\alpha$ acts by the multiplication with an eigenvalue $\xi$ on $H^{3,0}(X)$. Since $\alpha$ fixes $H^{3}(X, \mathbb{R})$, it acts by $\bar{\xi}$ on $H^{0,3}(X)$. Moreover $\alpha$ respects the polarization on $X$. Thus one has also that $\alpha$ acts by $\xi^{-1}$ on $H^{0,3}$. Hence

$$
\xi^{-1}=\bar{\xi} \Leftrightarrow|\xi|=1
$$

Since the centralizer $C_{S}(\alpha)$ of the action of $\alpha$ on $H_{S}^{3}(X, \mathbb{Q})$ is $\mathbb{Q}$-rational and contains $h_{S}\left(S^{1}\right)$, one has $\operatorname{Hg}\left(H_{S}^{3}(X, \mathbb{Q}), h_{S}\right) \subseteq C_{S}(\alpha)$. Therefore the action of $\alpha$ yields an element of $\operatorname{End}_{\mathbb{Q}}\left(H_{S}^{3}(X, \mathbb{Q}), h_{S}\right)$ and $\xi$ is contained in the number field $\mathbb{F}$ (see Lemma 1.3). Thus $\xi$ is a root of unity.

\section{The ACTIONS OF AUTOMORPhisms ON COMPLEX STRUCTURES}

Let $\alpha$ be an automorphism of $X$ and $\mathcal{X} \rightarrow B$ denote the Kuranishi family with $\mathcal{X}_{0}=X$ for $0 \in B$. In this section we consider the induced action of $\alpha$ on the Kuranishi family.

Definition 2.1. The automorphism $\alpha$ of $X$ is maximal, if it extends to an automorphism of $\mathcal{X}$ over its basis $B$.

A family or deformation $\mathcal{Y} \rightarrow \mathcal{Z}$ of $X$ is maximal, if the Kodaira-Spencer map is an isomorphism at every point.

Proposition 2.2. Let $\mathcal{Z}$ be a contractible complex manifold, $\mathcal{Y} \rightarrow \mathcal{Z}$ be a smooth holomorphic family of Calabi-Yau 3-manifolds with locally injective period map, $X=\mathcal{Y}_{0}$ and $\gamma$ be a diffeomorphism of $\mathcal{Y}$ over $\mathcal{Z}$ such that $\left.\gamma\right|_{X}=\mathrm{id}_{X}$. Then $\gamma$ is holomorphic.

Proof. Let $\gamma$ be a diffeomorphism of $\mathcal{Y}$ over $\mathcal{Z}$ with $\left.\gamma\right|_{X}=\operatorname{id}_{X}$. Hence for all $z \in \mathcal{Z}$ the restriction $\left.\gamma\right|_{Y_{z}}$ is isotopic to id. The cohomology $H^{3}\left(\mathcal{Y}_{z}, \mathbb{Z}\right)$ can be given by a Cech complex with respect to a finite covering

$$
\mathcal{U}=\left\{U_{i} \mid i=1, \ldots, N\right\}
$$

of contractible open sets. There exists a covering $\mathcal{U}^{\prime}$ of smaller contractible open subsets $U_{1}^{\prime}, \ldots U_{N}^{\prime}$ such that $\bar{U}_{i}^{\prime} \subset U_{i}$. On an open neighborhood $V$ of 0 one has $\bar{U}_{i}^{\prime} \subset\left(\left.\gamma\right|_{\mathcal{Y}_{z}}\right)\left(U_{i}\right)$. Thus $\gamma$ fixes the the Cech cohomology $H^{3}\left(\mathcal{Y}_{z}, \mathbb{Z}\right)$ on $V$. By the same argument, each $z_{0} \in \mathcal{Z}$ has a neighborhood $V$ such that the action of $\gamma$ on $H^{3}\left(\mathcal{Y}_{z}, \mathbb{Z}\right)$ coincides with the action of $\gamma$ on $H^{3}\left(\mathcal{Y}_{z}, \mathbb{Z}\right)$ for all $z \in V$. Thus the set of $z \in \mathcal{Z}$ such that $\gamma$ acts as id on $H^{3}\left(\mathcal{Y}_{z}, \mathbb{Z}\right)$ and its complement are both open. Since $\mathcal{Z}$ is connected and $\gamma$ acts as id on $H^{3}\left(\mathcal{Y}_{0}, \mathbb{Z}\right)$, the cohomology $H^{3}\left(\mathcal{Y}_{z}, \mathbb{Z}\right)$ is globally fixed by $\gamma$. This implies that the pullback preserves the Hodge structure of each $\mathcal{Y}_{z}$. Recall that the Kuranishi family has an injective period map (see [17], Lemma 1.5). Since the period map of $\mathcal{Y}$ is locally injective, the family is locally given by an embedding into the Kuranishi family. Hence the complex structure of the total space $\mathcal{Y}$ is also fixed by $\gamma$. Thus $\gamma$ is holomorphic. 
Proposition 2.3. For each automorphism $\alpha: X \rightarrow X$ there exists an open subset $B^{\prime} \subset B$ such that $0 \in B^{\prime}$ and the commutative diagram

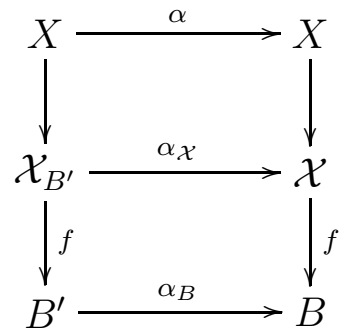

where the horizontal arrows represent biholomorphic maps and

$$
\alpha_{\mathcal{X}}=\left(\alpha, \alpha_{B}\right) \text { with respect to the trivialization } \mathcal{X}_{B^{\prime}} \cong X \times B^{\prime} .
$$

Proof. An automorphism $\alpha$ of a complex manifold is a diffeomorphism, which commutes with the almost complex structure induced from the complex structure. The complex structure of $\mathcal{X}$ diffeomorphic to $X \times B$ is given by an integrable almost complex structure $J$ on $X \times B$.

Since $\alpha \circ \alpha^{-1}=\mathrm{id}$, one concludes $d\left(\alpha^{-1}\right)=(d \alpha)^{-1}$. Hence

$$
\alpha_{\mathcal{X}}^{\prime}=(\alpha, \mathrm{id}):(X \times B, J) \rightarrow\left(X \times B, d \alpha_{\mathcal{X}}^{\prime} \circ J \circ d\left(\alpha_{\mathcal{X}}^{\prime}\right)^{-1}\right)
$$

is an almost complex map. Since $d \alpha_{\mathcal{X}}^{\prime} \circ J \circ d\left(\alpha_{\mathcal{X}}^{\prime}\right)^{-1}$ is also integrable $11 \alpha_{\mathcal{X}}^{\prime}$ can be considered as a holomorphic map with respect to the two complex structures.

Note that $\alpha_{\mathcal{X}}^{\prime}$ fixes the tangent directions with respect to $B$ in $X \times B$. Thus the projection onto $B$ is also holomorphic with respect to $d \alpha_{\mathcal{X}}^{\prime} \circ J \circ d\left(\alpha_{\mathcal{X}}^{\prime}\right)^{-1}$.

The Kuranishi family has the universal property that each deformation $\mathcal{Z} \rightarrow \mathcal{Y}$ of $X=\mathcal{Z}_{0}$ can be obtained by a holomorphic map $g: \mathcal{Y} \rightarrow B$ such that $\mathcal{Z}$ is the pullback of $\mathcal{X}$ with respect to $g$ over a neighborhood of $0 \in \mathcal{Z}$. Thus we have the open set $B^{\prime} \subset B$ with $0 \in B^{\prime}$ and the holomorphic map $g: B^{\prime} \rightarrow B$ such that the holomorphic family $\left(X \times B^{\prime}, d \alpha_{\mathcal{X}}^{\prime} \circ J \circ d\left(\alpha_{\mathcal{X}}^{\prime}\right)^{-1}\right)$ is given by the pullback with respect to $g$. Hence

$$
J=d g \circ d \alpha_{\mathcal{X}}^{\prime} \circ J \circ d\left(\alpha_{\mathcal{X}}^{\prime}\right)^{-1} \circ d g^{-1} .
$$

By $\alpha_{B}:=g$ and $\alpha_{\mathcal{X}}:=(\alpha, g)$, we obtain the desired holomorphic maps with respect to the original complex structure $J$. It is easy to see that the diagram commutes.

Remark 2.4. Let $B_{\alpha}$ be the subspace of $B^{\prime}$ on which $\alpha_{B}$ acts by id. We can choose coordinates on $B$ such that $0 \in B$ is given by $0 \in \mathbb{C}^{h^{2,1}(X)}$ and $B^{\prime}$ is given by an open neighborhood of $0 \in \mathbb{C}^{h^{2,1}(X)}$. Thus $\alpha_{B}$ is given by

$$
\alpha\left(b_{1}, \ldots, b_{h^{2,1}(x)}\right)=\left(\alpha_{1}\left(b_{1}, \ldots, b_{h^{2,1}(x)}\right), \ldots, \alpha_{h^{2,1}(X)}\left(b_{1}, \ldots, b_{h^{2,1}(x)}\right)\right) .
$$

One has that $\alpha$ fixes $\left(b_{1}, \ldots, b_{h^{2,1}(x)}\right) \in B^{\prime}$, if and only if

$$
\alpha_{1}\left(b_{1}, \ldots, b_{h^{2,1}(x)}\right)-b_{1}=\ldots=\alpha_{h^{2,1}(X)}\left(b_{1}, \ldots, b_{h^{2,1}(x)}\right)-b_{h^{2,1}(X)}=0 .
$$

Thus $B_{\alpha}$ is a complex analytic subspace of $B^{\prime}$. By Proposition 2.2 , the space $B_{\alpha}$ depends only on $\alpha$ and not on the choice of the trivialization. Moreover Proposition 2.2 tells us that an extension of a maximal automorphism $\alpha$ of $X$ can be given by $(\alpha$, id) with respect to each diffeomorphic trivialization $X \times B$.

Lemma 2.5. Let $\xi$ be the eigenvalue of the action of $\alpha$ on $H^{3,0}(X)$ and $B_{\alpha}$ have the dimension $r$. Then the eigenspace on $H^{2,1}(X)$ with eigenvalue $\xi$ of the action of $\alpha$ has at least the dimension $r$.

\footnotetext{
${ }^{1}$ Note that we have the same manifold and the same almost complex structure up to a diffeomorphism.
} 
Proof. Over $B_{\alpha}$ the automorphism $\alpha$ extends by $(\alpha$,id) with respect to the trivialization $X \times B_{\alpha}$. Assume without loss of generality that $0 \in B_{\alpha}$ is contained in the smooth locus of $B_{\alpha}$. Since $B_{\alpha}$ has the dimension $r$, the tangent space $T_{0} B_{\alpha}$ is generated by a basis

$$
\frac{\partial}{\partial x_{1}}, \ldots, \frac{\partial}{\partial x_{r}} \text {. }
$$

One is not able to transform different eigenspaces of an automorphism of a vector space smoothly. Since for each $p \in B_{\alpha}$ the space $F^{3}\left(\mathcal{X}_{p}\right)$ has to be contained in some eigenspace, one concludes that the bundle $F^{3}\left(\mathcal{X}_{B_{\alpha}}\right)$ is contained in the eigenspace with respect to some fixed eigenvalue $\xi$. Thus for a holomorphic section $\omega$ of $F^{3}(\mathcal{X})$ with $\omega(0) \neq 0$

$$
\frac{\partial \omega}{\partial x_{1}}(0), \ldots, \frac{\partial \omega}{\partial x_{r}}(0)
$$

are also contained in this eigenspace. Since

$$
\frac{\partial \omega}{\partial x_{1}}(0) \bmod F^{3}(X), \quad \cdots \quad \frac{\partial \omega}{\partial x_{r}}(0) \bmod F^{3}(X) \in H^{2,1}(X)
$$

are linearly independent, one gets the result.

Corollary 2.6. Let $\xi$ be the eigenvalue of the action of $\alpha$ on $H^{3,0}(X)$. Then $\alpha$ acts by the multiplication with $\xi$ on $H^{2,1}(X)$ and by the multiplication with $\xi^{-1}$ on $H^{1,2}(X)$ and $H^{0,3}(X)$, if $\alpha$ extends to an automorphism of the universal deformation $\mathcal{X} \rightarrow B$.

\section{The Cohomology aCtions of maximal AUtomorphisms}

3.1. Let us start with a not necessarily maximal automorphism $\alpha$, which is not a supertranslation and satisfies that $\alpha^{p}$ is a translation for some prime number $p>2$. The characteristic polynomial of the action of $\alpha$ on $H^{3}(X, \mathbb{Q})$ divides

$$
x^{p}-1=(x-1)\left(x^{p-1}+\ldots x+1\right) .
$$

Thus

$$
H^{3}(X, \mathbb{Q})=\operatorname{Eig}_{\mathbb{Q}}(\alpha, 1) \oplus N^{3}(X, \mathbb{Q}),
$$

where the action of $\alpha$ on $N^{3}(X, \mathbb{Q})$ yields an automorphism with characteristic polynomial $x^{p-1}+\ldots x+1$. Let $\xi$ be a primitive $p$-th. root of unity and consider

$$
N^{3}(X, \mathbb{Q}(\xi))=N^{3}(X, \mathbb{Q}) \otimes \mathbb{Q}(\xi) .
$$

The action of $\alpha$ yields a decomposition of $N^{3}(X, \mathbb{Q}(\xi))$ into the eigenspaces $\operatorname{Eig}_{\mathbb{Q}(\xi)}\left(\alpha, \xi^{r}\right)$ with $r=1, \ldots, p-1$. Let $\gamma \in \operatorname{Gal}(\mathbb{Q}(\xi), \mathbb{Q})$. Since the action of $\alpha$ on $H^{3}(X, \mathbb{Q}(\xi))$ is defined by rational matrices, $\alpha$ and $\gamma$ commute. Hence for $v \in \operatorname{Eig}_{\mathbb{Q}(\xi)}\left(\alpha, \xi^{r}\right)$ one has

$$
\gamma\left(\xi^{r}\right) \gamma(v)=\gamma\left(\xi^{r} v\right)=(\gamma \circ \alpha)(v)=\alpha(\gamma(v)) .
$$

Thus all eigenspaces $\operatorname{Eig}_{\mathbb{Q}(\xi)}\left(\alpha, \gamma\left(\xi^{r}\right)\right)$ have the same dimension $d$. Since there are $p-1$ primitive $p$-th. roots of unity, one has

$$
\operatorname{dim} N^{3}(X, \mathbb{Q})=d \cdot(p-1) .
$$

Lemma 3.2. Assume that $\alpha$ is not a super-translation and $\alpha^{p}$ is a translation for some prime number $p$. Moreover assume that $\alpha$ is maximal. Then $p=3$.

Proof. By Corollary 2.6, we have

$$
d=\operatorname{dim} F^{2}(X)=\frac{b_{3}(X)}{2} \text { and } \operatorname{Eig}_{\mathbb{Q}}(\alpha, 1)=0 .
$$

Thus:

$$
\frac{b_{3}(X)}{2}=d=\frac{b_{3}(X)}{p_{5}-1} \Rightarrow p=3
$$


By Lemma 1.4, each automorphism $\alpha$ acts by a root of unity on $H^{3,0}(X)$. Thus we conclude from Corollary 2.6 that each maximal automorphism $\alpha$ acts by the same root of unity on $F^{2}(X)$. Therefore each maximal automorphism $\alpha$ has a smallest positive integer $m$ such that $\alpha^{m}$ is a translation.

Corollary 3.3. Assume that the maximal automorphism $\alpha$ is not a translation. Then there exists an $m$, which has only the prime divisors 2 and 3 , such that $\alpha^{m}$ is a translation.

Assume that 9 is the smallest positive integer $m$ such that $\alpha^{m}$ is a translation. Then the characteristic polynomial divides

$$
x^{9}-1=\left(x^{6}+x^{3}+1\right)\left(x^{2}+x+1\right)(x-1)
$$

and there exists a subspace $N^{3}(X, \mathbb{Q}) \subset H^{3}(X, \mathbb{Q})$ such that the restriction of the action of $\alpha$ to $N^{3}(X, \mathbb{Q})$ has the characteristic polynomial $x^{6}+x^{3}+1$. By the same arguments as in 3.1, the vectorspace $N^{3}(X, \mathbb{Q})$ decomposes into 6 eigenspaces with the same dimension with respect to the 6 primitive 9-th. roots unity. Thus there does not exist a maximal automorphism $\alpha$ with $m=9$. Moreover there are 4 primitive 8 -th. roots of unity and 4 primitive 12-th. roots of unity. Thus we conclude:

Theorem 3.4. The group of maximal automorphisms of $X$ is up to translations given by

$$
\{e\}, \mathbb{Z} /(2), \mathbb{Z} /(3), \mathbb{Z} /(4) \text { or } \mathbb{Z} /(6) \text {. }
$$

\section{The construction of examples}

In this section let $B$ denote the moduli space of marked $K 3$ surfaces with universal family $\mathcal{S} \rightarrow B$ (see [2]). Moreover let $L$ be the integral cohomology lattice of a $K 3$ surface, $\phi$ denote an isometry of order 3 on $L$ and $\left(L_{\mathbb{C}}\right)_{\eta}$ denote the eigenspace on $L_{\mathbb{C}}$ with eigenvalue $\eta$ with respect to $\phi$. We fix

$$
\xi=\exp \left(\frac{2 \pi i}{3}\right) \text { and } r=\operatorname{dim}\left(L_{\mathbb{C}}\right)_{\xi}-1 .
$$

Construction 4.1. Assume that $\phi$ has a corresponding non-symplectic automorphism $\alpha$ on at least one $K 3$ surface. By the same arguments as in the proof of Proposition 2.3, the diffeomorphism $\alpha$ translates the complex structure on the universal family and yields a holomorphic map $\alpha_{B}: B \rightarrow B$ such that

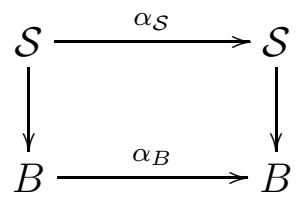

is a commutative diagram of holomorphic maps. Let $\Delta_{B}$ denote the diagonal. The intersection

$$
\emptyset \neq B_{\alpha}=\operatorname{Graph}\left(\alpha_{B}\right) \cap \Delta_{B}
$$

is an analytic subset. Thus we get the restricted family $\mathcal{S}_{\alpha} \rightarrow B_{\alpha}$ with a non-symplectic $B_{\alpha}$-automorphism of degree 3. Since the automorphism of each fiber $S$ corresponds to $\phi$, one has $H^{2,0}(S) \subset\left(L_{\mathbb{C}}\right)_{1}^{\perp}$. Assume without loss of generality that $H^{2,0}(S) \subset\left(L_{\mathbb{C}}\right)_{\xi^{-1}}$. The intersection form yields a Hermitian form of type $(1, r)$ on $\left(L_{\mathbb{C}}\right)_{\xi^{-1}}$. Thus the possible choices of $H^{0,2}(S)$ are given by the points of the corresponding ball $\mathbb{B}_{r}$ and the period map of $\mathcal{S}_{\alpha}$ is a locally injective map into $\mathbb{B}_{r}$. Since each point of $\mathbb{B}_{r}$ yields an up to a scalar unique vector $\omega$ with

$$
\omega \cdot \omega=0 \underset{6}{\operatorname{and}} \omega \cdot \bar{\omega}>0
$$


this ball is contained in the period domain of $K 3$ surfaces. Thus the period map into $\mathbb{B}_{r}$ is locally bijective.

Theorem 4.2. For $0 \leq k \leq 6$ one has a non-symplectic automorphism of degree 3 of a $K 3$ surface with a fixed locus consisting of $k$ disjoint smooth rational curves and $k+3$ isolated fixed points. One has that

$$
r=6-k \text {. }
$$

Proof. (see [1], [16])

Remark 4.3. In [5], Theorem 3.3 an example with $k=6$ has been constructed. An example of a family with $k=3$ occurs in [8], Section 12 and [14, Chapter 8. One finds an explicitly constructed fiber for $k=1$ in [15].

Construction 4.4. Let $\mathbb{E}$ denote the Fermat curve of degree 3 and $\alpha_{\mathbb{E}}$ denote a degree 3 automorphism of $\mathbb{E}$ acting by $\xi$ on $H^{1,0}(\mathbb{E})$. The quotient $\mathcal{S}_{\alpha} \times \mathbb{E} /\left\langle\left(\alpha_{\mathcal{S}}, \alpha_{\mathbb{E}}\right)\right\rangle$ is birationally equivalent to a family $\mathfrak{X}_{\alpha} \rightarrow B_{\alpha}$ of Calabi-Yau 3-manifolds. This construction method has been studied in [5], Proposition 3.1 and [14], Section 9.2.

From their actions on $H^{2,0}(S)$ and $H^{1,0}(\mathbb{E})$ one concludes that $\alpha_{\mathbb{E}}$ acts by $\xi$ and $\alpha_{\mathcal{S}}$ acts by $\left(\xi^{2}, 1, \ldots, 1\right)$ and $(\xi, \xi, 1, \ldots, 1)$ near their respective fixed loci. The singularities of $\mathcal{S}_{\alpha} \times \mathbb{E}_{3} /\left\langle\left(\alpha_{\mathcal{S}}, \alpha_{\mathbb{E}_{3}}\right)\right\rangle$ consist of families of curves and sections over $B_{\alpha}$. The singular sections can be resolved by blowing up the fixed sections over them before the application of the quotient map. In the case of the singularities given by families of curves the action of $\left\langle\left(\alpha_{\mathcal{S}}, \alpha_{\mathbb{E}}\right)\right\rangle$ is given by $\left(\xi, \xi^{2}, 1, \ldots, 1\right)$ near the corresponding fixed families of curves. We blow up the fixed families of curves with exceptional divisor $E_{1}$ and in a second step we blow up the families of fixed curves contained in $E_{1}$ with exceptional divisor $E_{2}$. Let

$$
\widetilde{\mathcal{S}_{\alpha} \times \mathbb{E}_{3}} \rightarrow B_{\alpha}
$$

denote the family obtained by the previous blowing up transformations. We have the quotient map

$$
\psi: \widetilde{\mathcal{S}_{\alpha} \times \mathbb{E}_{3}} \rightarrow \tilde{\mathfrak{X}}_{\alpha}:=\widetilde{\mathcal{S}_{\alpha} \times \mathbb{E}_{3}} /\left\langle\left(\alpha_{\mathcal{S}}, \alpha_{\mathbb{E}}\right)\right\rangle
$$

such that $\tilde{\mathfrak{X}}_{\alpha}$ is smooth. By blowing down $\psi\left(\tilde{E}_{1}\right)$ to a family of curves, we obtain the crepant resolution.

Proposition 4.5. Assume that the codimension one fixed locus in $\mathcal{S}_{\alpha}$ with respect to $\alpha_{\mathcal{S}}$ consists of families of rational curves. Then our family $\mathfrak{X}_{\alpha} \rightarrow B_{\alpha}$ is maximal.

Proof. Note that

$$
H^{3,0}\left(\mathfrak{X}_{p}\right)=H^{2,0}\left(\mathcal{S}_{p}\right) \otimes H^{1,0}(\mathbb{E})
$$

for each $p \in B_{\alpha}$. Moreover the period map of the family $\mathcal{S}_{\alpha}$ is a locally bijective map to the ball $\mathbb{B}_{r}$. Since $\mathbb{E}$ is a fixed curve with fixed Hodge structure, the period map of $\mathcal{S}_{\alpha}$ yields the period map of $\mathfrak{X}_{\alpha}$. Thus the period map of $\mathfrak{X}_{\alpha}$ is a locally bijective map to the ball $\mathbb{B}_{r}$. By the fact that we only blow up and down $\mathbb{P}^{1}$-bundles over families of rational curves and $\mathbb{P}^{2}$-bundles over sections, the desingularization does not have any effect on the third cohomology. Since

one concludes that

$$
b_{1}\left(\mathcal{S}_{p}\right)=b_{3}\left(\mathcal{S}_{p}\right)=0
$$

$$
\begin{aligned}
& H^{3}\left(\mathfrak{X}_{p}, \mathbb{C}\right)=H^{3}\left(\mathcal{S}_{p} \times \mathbb{E}, \mathbb{C}\right)_{1} \\
& =H^{1,0}(\mathbb{E}) \otimes\left(H^{2,0}\left(\mathcal{S}_{p}\right) \oplus H^{1,1}\left(\mathcal{S}_{p}\right)_{\xi^{2}}\right) \quad \oplus \quad H^{0,1}(\mathbb{E}) \otimes\left(H^{0,2}\left(\mathcal{S}_{p}\right) \oplus H^{1,1}\left(\mathcal{S}_{p}\right)_{\xi}\right)
\end{aligned}
$$

Thus

$$
h^{2,1}(\mathfrak{X})=h^{1,1}\left(\mathcal{S}_{p}\right)_{\xi^{2}}=r,
$$

which implies that $\mathfrak{X}_{\alpha}$ is maximal. 
Proposition 4.6. Our family $\mathfrak{X}_{\alpha} \rightarrow B$ has a degree 3 automorphism over its basis acting by $\xi$ on the $F^{2}$-bundle.

Proof. The automorphism $\alpha_{\mathbb{E}}$ acts on the family $\mathcal{S}_{\alpha} \times \mathbb{E}$ and commutes with $\left(\alpha_{\mathcal{S}}, \alpha_{\mathbb{E}}\right)$. On the exceptional divisor over the isolated fixed sections $\left(\alpha_{\mathcal{S}}, \alpha_{\mathbb{E}}\right)$ acts trivially. One can easily check that the actions of $\alpha_{\mathbb{E}}$ and $\left(\alpha_{\mathcal{S}}, \alpha_{\mathbb{E}}\right)$ are inverse to each other on $\tilde{E}_{1}$. Since $\left(\alpha_{\mathcal{S}}, \alpha_{\mathbb{E}}\right)$ acts trivially on $E_{2}$, the automorphism $\alpha_{\mathbb{E}}$ acts also on $\widetilde{\mathcal{S}_{\alpha} \times \mathbb{E}}$ and commutes with the induced action of $\left(\alpha_{\mathcal{S}}, \alpha_{\mathbb{E}}\right)$. Since $\alpha_{\mathbb{E}}$ fixes $\tilde{E}_{1}$, it descends to an automorphism of $\mathfrak{X}$. By the proof of Proposition 4.5, we have

$$
F^{2}\left(\mathfrak{X}_{p}\right)=H^{1,0}(\mathbb{E}) \otimes\left(H^{2,0}\left(\mathcal{S}_{p}\right) \oplus H^{1,1}\left(\mathcal{S}_{p}\right)_{\xi^{2}}\right) .
$$

Since $\alpha_{\mathbb{E}}$ acts by $\xi$ on $H^{1,0}(\mathbb{E})$ and the action of $\alpha_{\mathbb{E}}$ on $F^{2}\left(\mathfrak{X}_{p}\right)$ is given by the action on the corresponding differential forms on $\mathcal{S}_{p} \times \mathbb{E}$ via pullback, $\alpha_{\mathbb{E}}$ acts by $\xi$ on the $F^{2}$-bundle.

Proposition 4.7. The fibers of $\mathfrak{X}_{\alpha} \rightarrow B_{\alpha}$ are simply connected.

Proof. Let $M$ be a complex manifold. The fundamental group of $\mathbb{P}^{N}$ is trivial. Hence

$$
\pi_{1}(\tilde{M})=\pi_{1}(M)
$$

for each blowing up $\tilde{M}$ of a smooth curve or a point. Note that $K 3$ surfaces are simply connected and the fundamental group of $\mathcal{S}_{p} \times \mathbb{E}$ consists of $H_{1}(\mathbb{E}, \mathbb{Z})=H^{1}(\mathbb{E}, \mathbb{Z})^{*}$. Thus by the following Lemma, one obtains Proposition 4.7 .

Lemma 4.8. Let $f: X \rightarrow Y$ be a cyclic covering of manifolds of degree $n$, whose Galois group fixes at least one point $p \in X$ and does not fix any element $\pi_{1}(X, p) \backslash\{0\}$. Then

$$
\pi_{1}(Y)=0
$$

Proof. One can lift each path $\gamma$ on $Y$ with $\gamma(0)=\gamma(1)=f(p)$ to $n$ closed paths on $X$ with starting and ending point $p$. Each of these paths is mapped to $\gamma$. Thus the induced homomorphism $f_{*}: \pi_{1}(X) \rightarrow \pi_{1}(Y)$ is surjective. Since Gal $(f)$ does not fix any element of $\pi_{1}(X, p) \backslash\{0\}$, the induced homomorphism $f_{*}$ is the zero map.

Theorem 4.9. For $0 \leq k \leq 6$ one has a maximal family of simply connected Calabi-Yau 3 manifolds $X$ with a maximal automorphism acting by $\xi$ on $F^{2}(X)$. The Hodge numbers are given by

$$
h^{2,1}(X)=6-k \text { and } h^{1,1}(X)=18+11 \cdot k .
$$

Proof. By the previous results, it remains only to compute $h^{1,1}(X)$. Since each $K 3$ surface $S$ has the Betti numbers $b_{1}(S)=0$ and $b_{2}(S)=22$, one concludes that the eigenspace $h^{1,1}\left(\mathcal{S}_{p} \times \mathbb{E}\right)_{1}$ of $h^{1,1}\left(\mathcal{S}_{p} \times \mathbb{E}\right)$ with the eigenvalue 1 with respect to $\left(\alpha_{\mathcal{S}}, \alpha_{\mathbb{E}}\right)$ is given by:

$$
\begin{gathered}
h^{1,1}\left(\mathcal{S}_{p} \times \mathbb{E}\right)_{1}=h^{0,0}\left(\mathcal{S}_{p}\right) \cdot h^{1,1}(\mathbb{E})+h^{1,1}\left(\mathcal{S}_{p}\right)_{1} \cdot h^{0,0}(\mathbb{E}) \\
=22-2(r+1)+1=21-2 r
\end{gathered}
$$

For each isolated fixed section on $\mathcal{S}_{\alpha}$ we have to blow up 3 sections on $\mathcal{S}_{\alpha} \times \mathbb{E}$. Moreover for each fixed family of curves on $\mathcal{S}_{\alpha}$ we have altogether to blow up 9 families of curves and to blow down 3 families of curves. By Theorem 4.2, we have $0 \leq k \leq 6$ fixed families of curves, $n=k+3$ isolated fixed sections on $\mathcal{S}_{\alpha}$ and $r=6-k$. Thus we conclude:

$$
\begin{aligned}
h^{1,1}(X)= & h^{1,1}\left(\mathcal{S}_{p} \times \mathbb{E}\right)_{1}+6 \cdot k+3 \cdot n=21-2 r+6 \cdot k+3 \cdot(k+3) \\
& =21-12+2 \cdot k+6 \cdot k+3 \cdot k+9=18+11 \cdot k
\end{aligned}
$$




\section{Consequences for the monodromy}

Let $f: \mathcal{Y} \rightarrow \mathcal{Z}$ denote a maximal holomorphic family of Calabi-Yau 3-manifolds. Moreover assume that $X=\mathcal{Y}_{z}$ has a maximal automorphism $\alpha$.

Lemma 5.1. Let $\gamma:[0,1] \rightarrow \mathcal{Z}$ represent an element of $\pi_{1}(\mathcal{Z}, z)$. Then $\mathcal{Y}_{[0,1]}$ has the trivialization $X \times[0,1]$ and $(\alpha, \mathrm{id})$ yields for each fiber of $\mathcal{Y}_{\gamma([0,1])}$ a maximal automorphism.

Proof. Let $Z$ be the subset of $I=[0,1]$ consisting of points $p$ such that $(\alpha$, id) yields a maximal automorphism of $\mathcal{Y}_{\gamma(p)}$. Since $f: \mathcal{Y} \rightarrow \mathcal{Z}$ is maximal, $Z$ is open. Note that the complex structure of the Kuranishi family $\mathcal{X}$ of $\mathcal{Y}_{\gamma(p)}$ is given by an almost complex structure, i. e. a smooth section $J$ of $\operatorname{End}\left(T_{\mathcal{X}}\right)$. Thus the diffeomorphism $(\alpha$, id $)$ fixes the complex structure of any fiber of the local universal deformation of $\mathcal{Y}_{\gamma(p)}$, only if it fixes the complex structure of $\mathcal{Y}_{\gamma(p)}$. Now take $p \in \bar{Z}$. Thus $\alpha$ fixes also the complex structure on $\mathcal{Y}_{\gamma(p)}$. Therefore in a neighborhood $\mathcal{Z} \supset U_{p}$ of $\gamma(p)$ the fixed locus is given by

$$
\alpha_{1}\left(b_{1}, \ldots, b_{h^{2,1}(x)}\right)-b_{1}=\ldots=\alpha_{h^{2,1}(X)}\left(b_{1}, \ldots, b_{h^{2,1}(x)}\right)-b_{h^{2,1}(X)}=0
$$

(see Remark 2.4). Since $p \in \bar{Z}$, there is an open subset of $U_{p}$ on which all these holomorphic functions vanish. Thus these functions vanish on $U_{p}$ (see [18], Theorem 1.22). Hence $\bar{Z}=Z$ is a non-empty set, which is both closed and open. Since $I$ is connected, one concludes $Z=I$.

Theorem 5.2. Assume that $\alpha$ is not a super-translation. Then each matrix of the monodromy representation of $R^{3} f_{*}(\mathbb{C})$ fixes $F^{2}(X)$ and $\overline{F^{2}(X)}$ and is given by

$$
\left(\begin{array}{cc}
M & 0 \\
0 & \bar{M}
\end{array}\right)
$$

for some $\frac{b_{3}(X)}{2} \times \frac{b_{3}(X)}{2}$ matrix $M$ acting on $F^{2}(X)$.

Proof. Let $\xi$ denote the eigenvalue of the action of $\alpha$ on $H^{3,0}(X)$. Due to Lemma [5.1, the fiber $X=\mathcal{Y}_{\gamma(1)}$ has the maximal automorphism $\left.(\alpha, \mathrm{id})\right|_{X \times\{1\}}$. The diffeomorphism $\delta: \mathcal{Y}_{\gamma(1)} \rightarrow \mathcal{Y}_{\gamma(0)}=X$, which is holomorphic with respect to the respective complex structures induced by pullback, yields the monodromy action by push-forward. Thus the maximal automorphism $\rho(\gamma)(\alpha)$ of $X$ given by

$$
\rho(\gamma)(\alpha)=\left.\delta \circ(\alpha, \mathrm{id})\right|_{X \times\{1\}} \circ \delta^{-1}
$$

satisfies

$$
\operatorname{Eig}(\rho(\gamma)(\alpha), \xi)=\rho(\gamma)(\operatorname{Eig}(\alpha, \xi))
$$

Due to Corollary 2.6, both eigenspaces $\operatorname{Eig}(\rho(\gamma)(\alpha), \xi)$ and $\operatorname{Eig}(\alpha, \xi)$ coincide with $F^{2}(X)$. Thus the monodromy representation of $R^{3} f_{*}(\mathbb{C})$ fixes $F^{2}(X)$ and acts on $\overline{F^{2}(X)}$ by the complex conjugate matrix.

5.3. In literature [4], 9] one finds a formulation of the mirror conjecture based on the article [12]. For this formulation one assumes that the monodromy representation has some unipotent matrices $T_{1}, \ldots, T_{k}$ and defines the matrix $N$ by a linear combination of logarithms of $T_{1}, \ldots, T_{k}$. By using $N$, one defines a weight filtration

$$
0 \subset W_{0} \subseteq W_{1} \subseteq \ldots \subseteq W_{6}=H^{3}(X, \mathbb{Q}) \text { with } W_{0}=\operatorname{Im}\left(N^{3}\right) \text { and } \operatorname{dim} W_{0}=1 .
$$

Now assume that we are in the situation of Theorem [5.2. In this case $N^{3}$ acts by $M$ on $F^{2}(X)$ and by $\bar{M}$ on $\overline{F^{2}(x)}$. Thus $v \in F^{2}(X)$ is contained in $\operatorname{Im}\left(N^{3}\right)$, if and only if $\bar{v}$ is also contained in $\operatorname{Im}\left(N^{3}\right)$. Hence $\operatorname{Im}\left(N^{3}\right)$ has an even dimension. But 1 is not an even number. Therefore in the case of Theorem 5.2 the assumptions of this formulation of mirror symmetry can not be satisfied. 


\section{Consequences for COMPlex multiplication}

In this section we proof the following theorem:

Theorem 6.1. Assume that $X$ has a maximal automorphism $\alpha$, which is not a supertranslation. Then each maximal family over a connected basis, which contains $X$ as fiber, has a dense set of $C M$ fibers.

Recall that a Calabi-Yau 3-manifold $X$ has $C M$, if $\operatorname{Hg}\left(H^{3}(X, \mathbb{Q}), h_{X}\right)$ is a torus algebraic group. Calabi-Yau 3-manifolds with $C M$ are characterized by the following proposition:

Proposition 6.2. A Calabi-Yau 3-manifold $X$ has $C M$, if and only if the Hodge groups of the weight one Hodge structures corresponding to $\mathrm{J}_{G}(X)$ and $\mathrm{J}_{W}(X)$ are tori and commute.2

Proof. (see [3], Theorem 2.3)

The proof of Theorem 6.1 follows arguments and ideas similar to [14], Section 4.3 and Section 4.4. We use the theory of Shimura varieties, which is explicateted in [6, [7]. The vector space automorphism $J$, which acts by $i$ on $F^{2}(X)$ and $-i$ on $\overline{F^{2}(X)}$, fixes $H^{3}(X, \mathbb{R})$. Thus $J$ is a complex structure on $H^{3}(X, \mathbb{R})$, i. e. $J^{2}=-$ id. By

$$
F^{2}(X) \rightarrow\left(H^{3}(X, \mathbb{R}), J\right), \quad v \rightarrow \tilde{v}=v+\bar{v}
$$

we have an isomorphism of complex vector spaces. The cup product yields an alternating form $Q$ on $H^{3}(X, \mathbb{Q})$. By $Q$, we get the Hermitian form

$$
H(\cdot, \cdot)=i Q(\cdot, \cdot)
$$

on $H^{3}(X, \mathbb{C})$ such that the Hodge decomposition is orthogonal with respect to $H$. Moreover let

$$
C(\alpha) \subset \operatorname{Sp}\left(H^{3}(X, \mathbb{Q}), Q\right)
$$

denote the centralizer of the action of $\alpha$ on $H^{3}(X, \mathbb{Q})$. Let for each $N \in \mathrm{GL}\left(F^{2}(X)\right)$ the matrix $\bar{N} \in \mathrm{GL}\left(\overline{F^{2}(X)}\right)$ be given by

$$
\bar{N} \bar{v}=\overline{N v} \quad\left(\forall v \in F^{2}(X)\right) .
$$

We obtain the isomorphism $t r: \mathrm{GL}\left(F^{2}(X)\right) \rightarrow \mathrm{GL}\left(H^{3}(X, \mathbb{R}), J\right)$ of $\mathbb{C}$-algebraic groups by

$$
\mathrm{GL}\left(F^{2}(X)\right) \ni N \rightarrow N \times \bar{N} \in \mathrm{GL}\left(F^{2}(X)\right) \times \mathrm{GL}\left(\overline{F^{2}(X)}\right) .
$$

Lemma 6.3.

$$
C(\alpha)(\mathbb{R})=\operatorname{tr}\left(\mathrm{U}\left(F^{2}(X),\left.H\right|_{F^{2}(X)}\right)(\mathbb{R})\right)
$$

Proof. Assume that $N \in \mathrm{U}\left(F^{2}(X),\left.H\right|_{F^{2}(X)}\right)(\mathbb{R})$. Then $\operatorname{tr}(N)$ fixes the two eigenspaces (compare Corollary 2.6). Thus it commutes with $\alpha$. By using (1), we get that each $N \in \mathrm{U}\left(F^{2}(X),\left.H\right|_{F^{2}(X)}\right)(\mathbb{R})$ satisfies that $\operatorname{tr}(N) \in \operatorname{Sp}\left(H^{3}(X, \mathbb{Q}), Q\right)(\mathbb{R})$. Thus

$$
\operatorname{tr}\left(\mathrm{U}\left(F^{2}(X),\left.Q\right|_{F^{2}(X)}\right)(\mathbb{R})\right) \subseteq C(\alpha)(\mathbb{R}) .
$$

Let $M \in C(\alpha)(\mathbb{R})$. Thus $M$ fixes the two eigenspaces $F^{2}(X)$ and $\overline{F^{2}(X)}$. Since $M \in \operatorname{Sp}\left(H^{3}(X, \mathbb{Q}), Q\right)(\mathbb{R})$, one obtains $M \in \operatorname{tr}\left(\mathrm{U}\left(F^{2}(X),\left.H\right|_{F^{2}(X)}\right)(\mathbb{R})\right)$ by using (1). Thus

$$
C(\alpha)(\mathbb{R}) \subseteq \operatorname{tr}\left(\mathrm{U}\left(F^{2}(X),\left.Q\right|_{F^{2}(X)}\right)(\mathbb{R})\right) .
$$

\footnotetext{
${ }^{2}$ Some authors write that a Calabi-Yau 3-manifold has $C M$, if and only if its Griffiths intermediate Jacobian has $C M$. For a proof they incorrectly quote the same article [3].
} 
Since $\alpha$ yields a Hodge isometry of $H^{3}(X, \mathbb{Z})$, one obtains

$$
h\left(S^{1}\right) \subset C(\alpha)_{\mathbb{R}}
$$

One checks easily that the adjoint representation yields the homomorphism

$$
h^{\text {ad }}: S^{1} \rightarrow C(\alpha)_{\mathbb{R}}^{\text {ad }} \cong \mathrm{PU}\left(1, h^{2,1}(X)\right) \text { given by } z \rightarrow\left(z, z^{-1}, \ldots, z^{-1}\right) .
$$

The centralizer of $h\left(S^{1}\right)$ in $\operatorname{Sp}\left(H^{3}(X, \mathbb{Q}), Q\right)$ coincides with the maximal compact subgroup $K$ of $\operatorname{tr}\left(\mathrm{U}\left(F^{2}(X),\left.Q\right|_{F^{2}(X)}\right)\right)$ isomorphic to $\mathrm{U}(1) \times \mathrm{U}\left(h^{2,1}(X)\right)$. Therefore the centralizer of $h^{\text {ad }}\left(S^{1}\right)$ in $C(\alpha)_{\mathbb{R}}^{\text {ad }}$ is a maximal compact subgroup isomorphic to $\mathrm{P}\left(\mathrm{U}(1) \times \mathrm{U}\left(h^{2,1}(X)\right)\right)$. Thus $\left(C(\alpha)^{\text {ad }}, h^{\text {ad }}\right)$ is a Shimura datum, which yields the complex ball $\mathbb{B}_{h^{2,1}(X)}$.

Since the $F^{2}$-bundle is rigid, the period map of the Kuranishi family $\mathcal{X} \rightarrow B$ is given by the fractional period map

$$
p_{F^{2}}: B \rightarrow \mathbb{P}\left(F^{2}(X)\right) .
$$

Since the period map of the Kuranishi family is injective (see [17], Lemma 1.5) and

$$
h^{2,1}(X)=\operatorname{dim} \mathbb{P}\left(F^{2}(X)\right)=\operatorname{dim} B,
$$

$p_{F^{2}}$ is open. Moreover each open subset of the period domain

$$
C(\alpha)^{\operatorname{ad}}(\mathbb{R}) / \operatorname{ad}(K)(\mathbb{R}) \cong \mathbb{B}_{h^{2,1}(X)}
$$

associated to the Shimura datum $\left(C(\alpha)^{\text {ad }}, h^{\text {ad }}\right)$ is given by an open set of $\mathbb{P}\left(F^{2}(X)\right)$. Thus we have an open map

$$
B \rightarrow C(\alpha)^{\operatorname{ad}}(\mathbb{R}) / \operatorname{ad}(K)(\mathbb{R}),
$$

which assigns to each point $p \in B$ the Hodge structure on $H^{3}\left(\mathcal{X}_{p}, \mathbb{Q}\right)$. Since the set of $C M$ points is dense in $C(\alpha)^{\text {ad }}(\mathbb{R}) / \operatorname{ad}(K)(\mathbb{R})$ (see [14], Theorem 1.5.9), one concludes:

Proposition 6.4. Assume that the Kuranishi family of $X$ has a maximal automorphism, which is not a super-translation. Then it has a dense set of $C M$ fibers.

By the same arguments as in the proof of Lemma 5.1, a maximal automorphism extends along each path in the basis of a maximal family. Thus one obtains Theorem 6.1.

\section{REFERENCES}

[1] Artebani, M., Sarti, A.: Non-symplectic automorphisms of order 3 on $K 3$ surfaces. (2008) arXiv:0801.3101.

[2] Barth, W., Hulek, K., Peters, C., Van de Ven, A.: Compact Complex Surfaces. Second Enlarged Edition, Ergebnisse der Mathematik und ihrer Grenzgebiete 3, Vol. 4, Springer-Verlag (2004) Berlin Heidelberg New York Tokyo.

[3] Borcea, C.: Calabi-Yau threefolds and complex multiplication. Essays on mirror manifolds. Internat. Press (1992) Hong Kong, 489-502.

[4] Cox, D., Katz, S.: Mirror Symmetry and Algebraic Geometry. Mathematical Surveys and Monographs 68, AMS (1999) USA.

[5] Cynk, S., Hulek, K.: Construction and examples of higher-dimensional modular Calabi-Yau manifolds. Canad. Math. Bull. 50, (2007) 486-503.

[6] Deligne, P.: Travaux de Shimura. In: Seminaire Bourbaki, 389 (1970/71), Lecture Notes in Mathematics 244, Springer-Verlag, Berlin (1971) 123-165.

[7] Deligne, P.: Variétés de Shimura: interprétation modulaire, et techniques de construction de modèles canoniques. In: Automorphic forms, representations and $L$-functions (Proc. Sympos. Pure Math., Oregon State Univ., Corvalis, Ore., 1977), Part 2, Proc. Sympos. Pure Math., XXXIII, AMS, Providence, R.I. (1979) 247-289.

[8] Dolgachev, I., Kondo, S.: Moduli spaces of K3 Surfaces and Complex Ball Quotients (2005) arXiv:0511051.

[9] Gross, M., Huybrechts, D., Joyce, D.: Calabi-Yau Manifolds and Related Geometries, SpringerVerlag (2003) Berlin Heidelberg New York. 
[10] Gukov, S., Vafa, C.: Rational Conformal Field Theories and Complex Multiplication. (2002) hep-th./0203213.

[11] Lang, S.: Complex Multiplication. Grundlehren der mathematischen Wissenschaften 255, Springer-Verlag (1983) Berlin Heidelberg New York.

[12] Morrison, D.: Compactifications of moduli spaces inspired by mirror symmetry. Journées de géométrie algébrique d'Orsay, Astérisque 218 (1993) 243-271.

[13] Nikulin, V. V.: Discrete Reflection Groups in Lobachevsky Spaces and Algebraic Surfaces. In: Proc. Intern. Cong. Math., Berkeley (1986) 654-669.

[14] Rohde, J. C.: Cyclic coverings, Calabi-Yau manifolds and Complex multiplication. (2007) arXiv:0711.2195, to appear in Lecture Notes in Mathematics 1975, Springer-Verlag (2009).

[15] Rohde, J. C.: Examples of Calabi-Yau 3-manifolds with Complex multiplication. (2008) arXiv:0803.2978, to appear as appendix of [14].

[16] Taki, S.: Classification of non-symplectic automorphisms of order 3 on $K 3$ surfaces. (2008) arXiv:0802.1956

[17] Voisin, C.: Variations of Hodges Structure of Calabi-Yau Threefolds. Publications of the Scuola Normale Superiore (1996) Rom.

[18] Voisin, C.: Théorie de Hodge et géométrie algébrique complexe. Cours spécialisés 10, SMF (2002) France.

GRK 1463 / Institut für Algebraische Geometrie, Leibniz Universität Hannover, 30060 HanNover, GeRmany

E-mail address: rohde@math.uni-hannover.de 